\title{
Three-Dimensional Facial Imaging using a Static Light Screen and a Dynamic Subject
}

\author{
Robert McKeon and Patrick Flynn \\ The University of Notre Dame \\ Computer Vision Research Lab \\ \{rmckeon, flynn\}@nd.edu
}

\begin{abstract}
Many commercially available $3 D$ sensors suitable for face image capture employ either passive (or textureassisted) stereo imaging or structured illumination with a moving stripe. Both of these techniques require a stationary subject. We describe an initial design and evaluation of a fixed-stripe, moving object $3 D$ scanner designed for human faces. Our method of acquisition requires the subject to walk through a light screen generated by two laser line projectors. Triangulation and tracking yield a 3D image of the subject's face from multiple images. To demonstrate the accuracy of our initial design, a small-scale facial recognition experiment was executed. In an experiment involving 13 subjects with 4 images per subject, we achieve 92.3\% rank one recognition using an Iterative Closest Point (ICP) based matching method, demonstrating the feasibility of the technique.
\end{abstract}

\section{Introduction}

Biometrics is a technology of increasing relevance to security applications in the private and public sectors. Facial recognition (in particular, 3-dimensional (3D) facial recognition) is a subfield of recent active research. 3D facial recognition (as opposed to 2D facial recognition) can exploit the near invariance of facial shape images to facial color variations and facial illumination changes. Triangulation is a popular method used to acquire a 3D image of an object at short range. The three main techniques for triangulation are stereo vision, structured light, and structure from motion.

Using structured light to acquire a 3D image of a subject's face is typically intrusive. In many cases, a subject has to remain motionless for a few seconds for the hardware to acquire an accurate 3D image of the subject's face. Our goal is to build a non-intrusive, 3D facial scanner capable of outputting images with a high enough quality to be used for facial recognition.

The traditional method for triangulation using active light translates a line or line pattern across an object (in our case, a face) and recovers a 3D profile at each position based on the deformation of the image of the line. This process involves calibration of both the line projector and the motion platform. By contrast, we employ a fixed light screen through which the subject moves. In this fashion, a camera also sees the deformation of the laser line yielding a profile, and we assemble a 3D image from multiple images by knowing this deformation and the subject's instantaneous velocity. We calibrate the cameras for a single, fixed plane of light and rely on subject motion to provide the multiple positions needed to acquire 3D face data.

\section{Previous Work}

The traditional method of triangulation using structured light involves one camera and one line projector set apart at different angles. Object occlusion is a major challenge involved in triangulation with this particular technique. If part of the object occludes the camera's view of the light stripe, the 3D image will have a hole in place of the occluded geometry. This particular problem can be seen in images from triangulation-based 3D face scanners around the nose and in concavities such as the eye sockets.

The two main steps in triangulation using structured light are acquisition of an image of the light stripe on the object and processing of the image to extract depth information. Techniques used for image acquisition involve varying camera and lighting arrangements while techniques for processing the images fall into two categories: spatial domain processing and temporal domain processing.

\subsection{Acquisition}

Previous 3D scanning systems have employed a single camera/light source pair with a variety of hardware to improve acquisition rates. Other researchers added multiple camera views (both real and imaginary) or added multiple light sources to improve coverage or image quality. Success was also found using light patterns or adding human interaction to improve the final 3D image. 
The PRIME 3D scanning system [1] uses one camera, one laser line projector, and a controlled conveyor belt to image different types of objects as seen in Figure 1. The encoder wheel provides displacement information of the object, and as the object passes through the light stripe, the camera acquires many images that are later aligned using the displacement information.

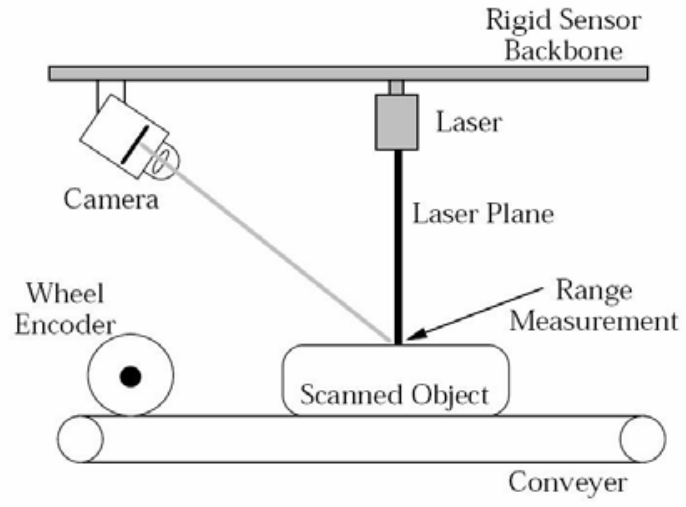

Figure 1: PRIME's range finding setup [1].

Many objects are too large to be scanned in this manner, like people, which is why other range finders have been developed using a moving stripe and VLSI chips specifically designed for high-speed acquisition [2, 3]. However, customized hardware is expensive.

Holes in the resulting range image caused by occlusions are a major problem with a single camera system. A sensor designed by Medioni and Jezouin [4] and Saint-Marc et al. [5] were similar to PRIME but included an additional camera to alleviate this problem. Saint-Marc et al. [5] constructed a 3D scanner with a static light screen and a linear table to move an object through their light screen while 2 cameras at different angles capture images of the laser line. This simplified calibration of their light screens since they did not move.

Davis and Chen [6] used 2 cameras and a rotary actuator to move a projected line across an object but highlighted the difficulty in calibrating their system for each position of the stripe, a common problem in structured lighting. They used the knowledge of the camera positions to determine the depth component of the pixels along the stripe without knowing the exact position of the rotary actuator. This technique worked to reduce the complexity of calibration.

More cameras may be added as in Pulli et al. [7] where four cameras were used to image an object turned on a rotary stage while a light pattern was projected. Griesser et al. [8] acquired full 3D images of dynamic objects using a four camera/projector setup to project the same light pattern onto the object from four different angles.

Davis and Chen [6] used mirrors to create imaginary cameras, and Lanman et al. [9] takes this idea further by using two mirrors to create four imaginary cameras. A light pattern is projected on the object, and due to the mirrors, five views of the object at different angles are used to construct a full 3D image. They only experimented with the imaging of smaller objects even though their idea could be easily scaled to a larger size.

Adding more light patterns can also help solve the occlusion problem, but stripe disambiguation becomes a problem in this case. Jalkio et al. [10] examined the problem of resolving stripe identity in a dual laser stripe system. They used a pair of symmetric lenses along with a triangle lens to insure that the images of both stripes were in focus and the stripes themselves were disjoint. Park et al. [11] also added an additional laser stripe so that their system resembled PRIME's with an extra laser. This filled in many occlusion holes. The intersection of the two light stripes was designed to be past the plane of the conveyor belt, thus resolving the ambiguity.

Wang and Pandey [12] used two sets of parallel line stripes to solve the occlusion problem in a $3 \mathrm{D}$ sensor designed for the tracking of dynamic 3D objects. There were failure cases if the object became occluded or if the separation between parallel lines was too small. Park and Kim [13] used a cone shaped light pattern to find seams in objects. This method was similar to employing more than one light plane due to the cone shape, but occlusion problems still occurred for larger objects.

Another problem related to such techniques is balancing the line count against the number of images needed for acquisition. In order to increase the line count, some have used colored stripes to provide a denser lighting structure whose individual lines can be disambiguated. Boyer and Kak [14] used a multi-colored grid pattern displayed on a target, and they were successful in separating the different color stripes for the purpose of structured light range sensing. Zhang et al. [15] used four colors and determined that the space between each colored line could be used to find depth points. Zhang et al. [16] used a high frequency pattern of colored stripes for triangulation but had difficulty with objects that were poor at reflecting color. Gu et al. [17] use the phase information for triangulation by displaying a frequency interference pattern across three color planes. Their technique was helpful in many areas but suffered from the same problem as other color techniques, which is that they fail when the colors are not visible.

Stripe pattern coding has been used to reduce the number of images one has to take using structured light to get a full range image. Lee et al. [18] used this technique to build a range finder by matching the received image of each pattern to the original pattern projected on the object. Tomita et al. [23] also used modulated patterns to reduce the number of images required to produce a range image. 
More elaborate 3D sensing systems like those proposed by Fisher el al. [19] and Borghese et al. [20] result in more complete 3D models, but human interaction is required. Fisher et al. [19] used a fixed light source and a moving wand to cast a shadow over the object for triangulation. By finding the edge of this shadow, they made a range image. Borghese et al. [20] used 2 cameras and one laser where the user moves the object through the laser plane, and the user can see the real-time 3D image on the screen to help them find parts of the image requiring more data due to occlusions. The technique used by Borghese et al. [20] is an application of ranging sensing in building accurate models. Both of these techniques require a large amount of manipulation of the object, so they would not be helpful techniques for facial imaging.

\subsection{Data Processing}

Traditionally, most methods similar to PRIME [1] find the center of the light stripe to subpixel accuracy in the spatial domain by assuming a Gaussian profile for the stripe cross-section. However, others have proposed using time domain analysis to find depth points for an image. This involves examining one pixel over time and estimating the time of arrival of a peak intensity value for that pixel. At that time, the center of the line is passing over that point. Images of the center of the line passing directly over a particular pixel may not have been acquired, but using temporal domain processing, the location of the peak of the line of a given pixel can be estimated with a high accuracy.

A few researchers have explored this idea at length. Curless and Levoy [21] point out the major problems with spatial domain processing and show how temporal domain processing can solve these problems. Davis et al. [22] integrate both spatial and temporal processing to allow for more detailed and robust triangulation techniques. Tomita et al. [23], Hall-Holt and Rusinkiewicz [24], and Pulli et al. [7] have used time processing techniques in their work to achieve more accurate range images.

\section{Method}

We built a system in which the light screen is fixed, and a human subject walks through it at a normal speed. Figure 2 depicts the sensing rig. We form the light screen using two laser stripe projectors set on opposite sides of the subject, aligned to be parallel with each other and vertically positioned at the same height. This screen corresponds to the $z=0$ plane of the sensor's output coordinate system. The light planes generated by these two projectors are thus coincident. Two cameras are used for triangulation, and a third camera generates images used to track the subject's velocity in the sensor's $z$ coordinate (perpendicular to the light screen). 3D data is acquired as the human subject walks through the light screen using time domain analysis. We use only a portion of the full camera image (386 x 224 instead of $480 \times 640$ ) to achieve a higher frame rate. This camera view can be considered an approximation of a pinhole camera in the sense that radial camera distortion is minimized as seen in Figure 5 which has obvious perspective distortion but not radial distortion.

We used two cameras with two lasers to provide greater coverage of the face. The two cameras used for triangulation acquire in color (RGB) and their image coordinates will be referred to as $\left(u_{1}, v_{1}\right)$ and $\left(u_{2}, v_{2}\right)$ where needed. The side camera acquires in grayscale; horizontal displacement in that camera is the only measurement of interest and will be denoted w. A sample of the images from each camera can be seen in Figure 4.

We used three high speed Firewire cameras to capture images, and two lasers with cylindrical lens to serve as stripe projectors (Figure 3). The intensity gain was adjusted so only the laser showed up in the image, as seen in Figure 4. While this improves detection of the laser line, the ability to detect facial color using the same camera is lost.

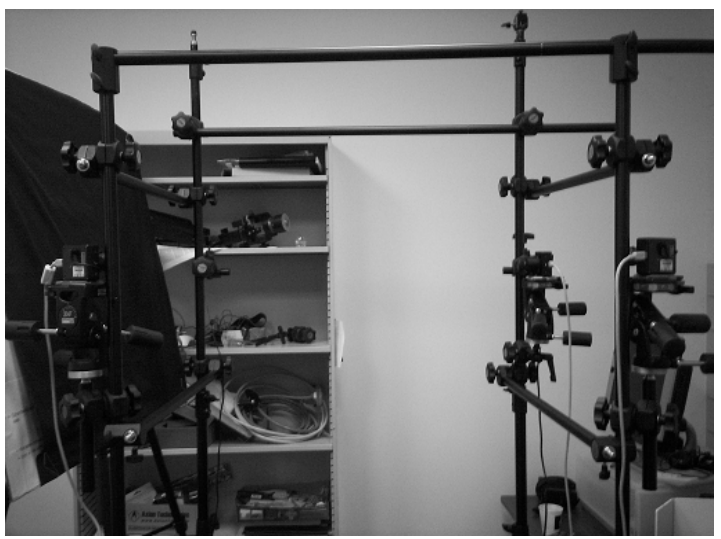

Figure 2: A picture of the 3D face imaging rig.

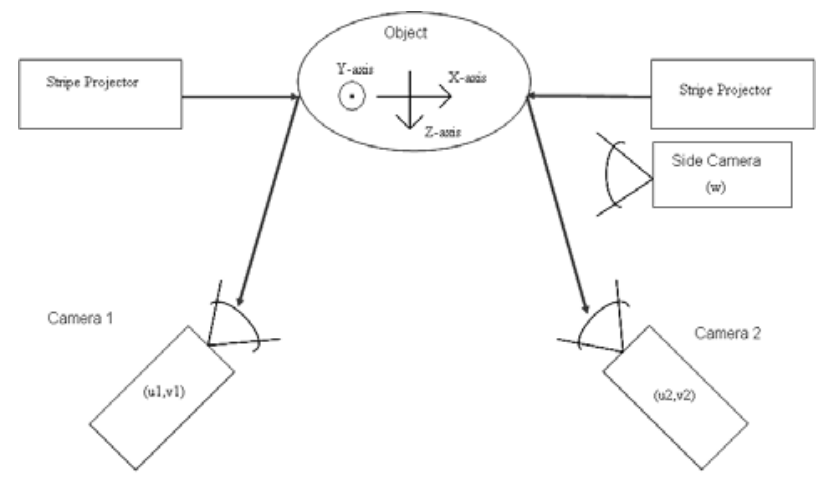

Figure 3: Experimental apparatus for 3D sensing of a dynamic object. 
(a)

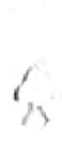

(f)

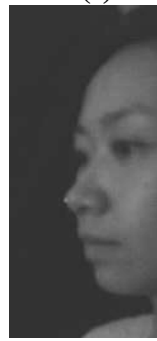

(k) (b)

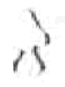

(c)

(d)

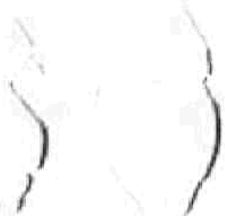

(e) most of the squares on the board, also shown in Figure 5. A position at any point within the trapezoid can be rectified by interpolating known values from the region corners. Thus, a measurement $\left(u_{i}, v_{i}\right)$ in either frontal camera's field of view maps via interpolation to stripe plane coordinates $\left(x_{i}, y_{i}\right)$.

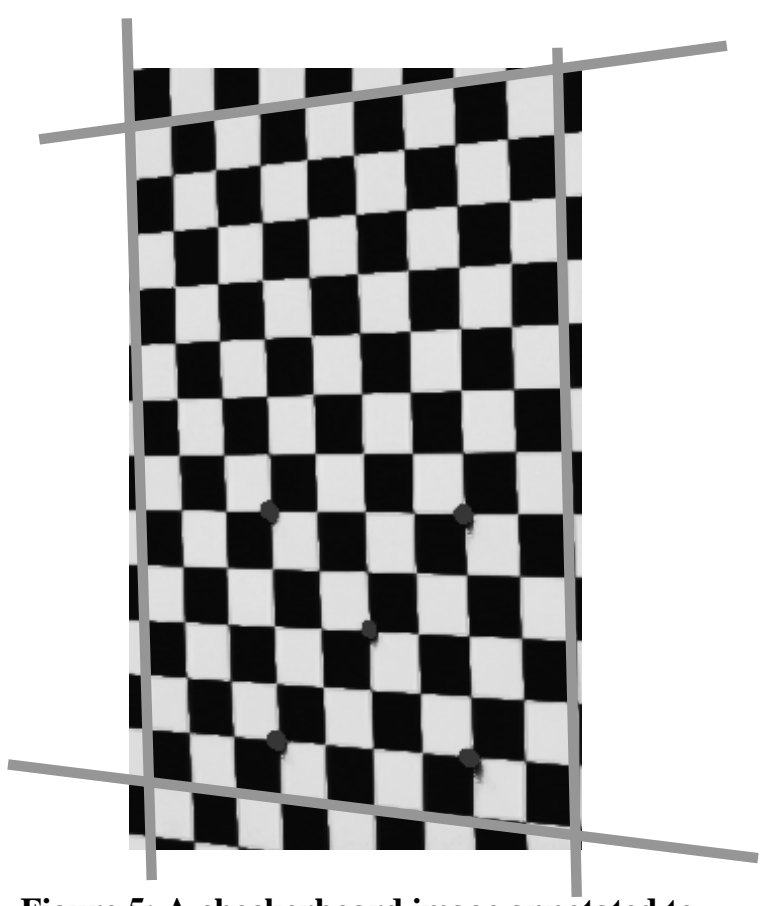

Figure 5: A checkerboard image annotated to show the boundary of a 9x12 square inch region to be rectified.

An image of the laser screen's intersection with the subject's face is formed for each frontal camera. The origin for 3D output coordinates is specified by a distinguished point on the calibration checkerboard, identified by a thumbtack.

\subsection{Side camera calibration and $z$-coordinate determination for the subject}

The side camera provides the $z$ displacement of the subject as they move through the light sheet, and thus a $z$ coordinate for each stripe acquired during movement. In the current version of our system, we assume that the head is aligned with the direction of travel and that no significant head rotation takes place during the acquisition of data. The nose position provides the subject's reference position as it is easily detected in a face profile. The camera acquires in grayscale with a low gain setting (Figure 6 (a)), and we remove most of the background noise using background subtraction (Figure 6 (b)). The side camera is calibrated using a checkerboard calibration chart, as presented earlier in the context of the frontal cameras. 
The nose position is found as follows. An image is acquired, the background is suppressed, and the image is mapped to $Z$ coordinates (Figure 6(c)) view. Lowdifference pixels are removed by thresholding. (Figure 6 (d)), we delete any connected components in this image containing fewer than 200 pixels leaving just the face (Figure 6 (e)). We define the farthest point to the left in the largest remaining connected component to be the nose as seen at the line in Figure 6 (f).

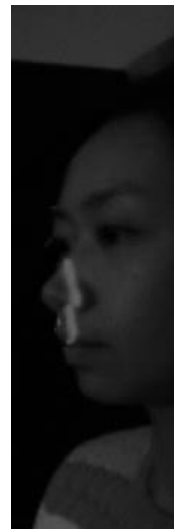

(a)

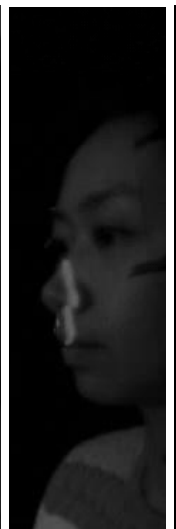

(b)

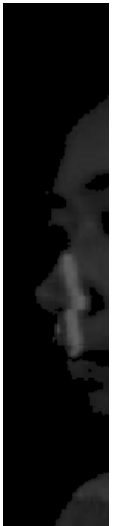

(c)

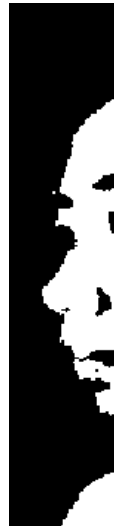

(d)

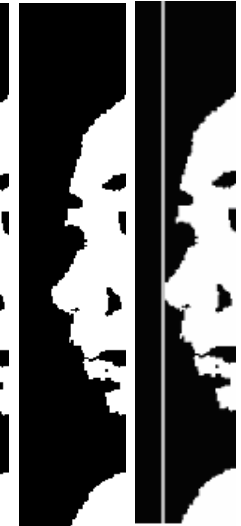

(e) (f)
Figure 6: (a) Original image. (b) Image after background suppression. (c) Image projected to world (z) coordinates. (d) World coordinate image after thresholding. (e) Result of small connected component removal. (f) The line shows the $z$ position of the nose being the farthest point to the left.

\subsection{Stripe detection}

Detection of the laser stripe in a frontal view is essentially finding pixels with maximal red values. The color of a human face resides mostly in the red and green planes, not the blue plane due to the saturation setting of the camera. By subtracting the green plane from the red plane, we isolate the laser line while deleting the background noise. Using this new intensity image, the maximum pixel intensity for each row of each image was found, and a threshold value was found by taking $15 \%$ of the average of these maximum pixel values. Then pixels of the new intensity image below this threshold were deleted. The threshold usually was found to be low enough to keep all the pixels belonging to the stripe, while high enough to suppress low intensity noise.

We find the center of the laser line in the image (the line's width is not constant due to the Gaussian profile of the stripe cross-section, whose spread increases with standoff) using temporal domain processing [20]. As a laser stripe passes over a given image pixel $\left(u_{i}, v_{i}\right)$ pixel, the intensity of the pixel can be assumed to sample a
Gaussian curve over time, as shown in Figure 7 (a). We obtain a subpixel estimate [25] of the peak as follows. The $z$ (i.e. time) value yielding the maximum intensity is found and denoted $z_{\max }$. We then compute the weighted sum of $z$ values within 5 pixels of $z_{\max }$, yielding a subpixel estimate $Z_{\text {peak }}$ :

$$
z_{\text {peak }}=\frac{\sum_{i=z_{\max }-5}^{z_{\max }+5} z_{i} \cdot I\left(u, v, z_{i}\right)}{\sum_{i=z_{\max }-5}^{z_{\max }+5} I\left(u, v, z_{i}\right)}
$$

where $I\left(u, v, z_{i}\right)$ is the intensity of the pixel at the $(\mathrm{u}, \mathrm{v})$ coordinate in the image at the time instance $z_{i}$.

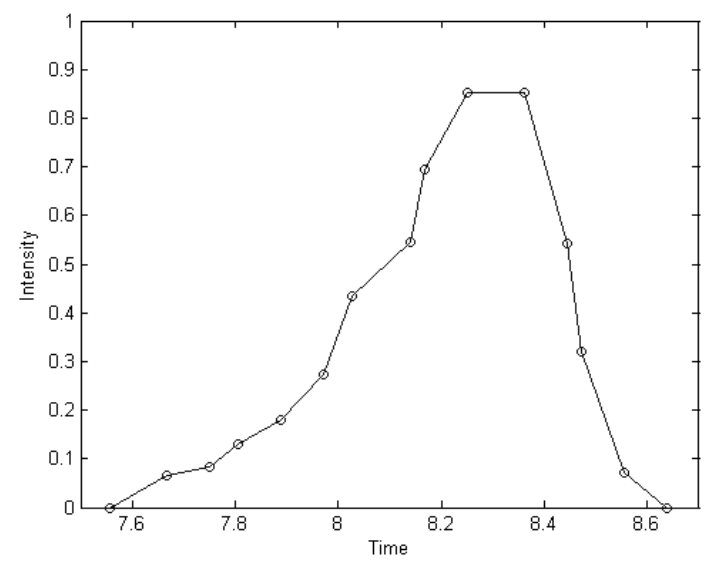

(a)

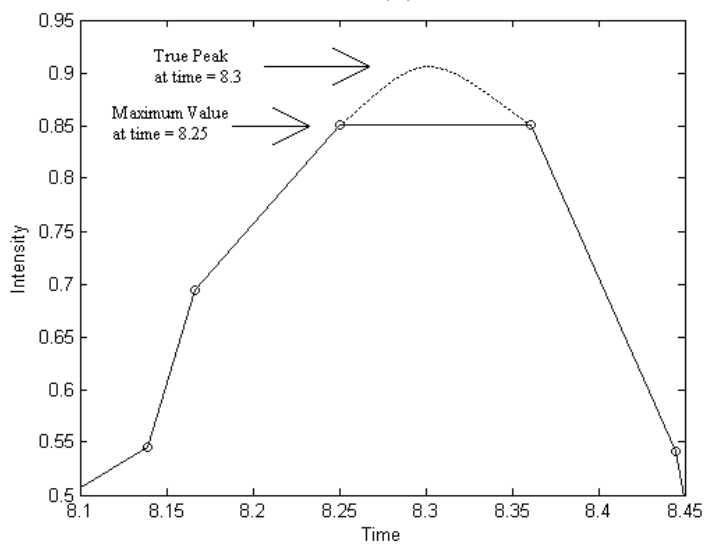

(b)

Figure 7: (a) an example of temporal domain stripe peak estimation. (b) Shows the peak of this example where the maximum value being found at time $t=8.25$, but the true peak found using the weighted average is at 8.3 .

Processing the data in the time domain has a maximum error of half the laser line's cross-sectional thickness, and this occurs at pixels that the stripe illuminated only once or twice during the run. A higher 
frame rate will reduce this error. We currently use 96 frames per second, but even at this high frame rate, a subject could walk through the sensor too quickly. At present, we ask subjects to proceed slowly through the screen.

By transforming of pixel coordinates to real coordinates using the calibration data, real world 3D was produced. By fusing the data front the two front cameras using the calibration offset, we created a 3D image by making a mesh using a Delaunay triangulation [26] as seen in Figure 8 (a). This figure shows a surface mesh on the data, and those parts of the surface mesh that were too large were deleted, thus the holes. One can see there are a few large holes, particularly by the nose on the cheek, below the nose, on the forehead, and on the chin.

The eye sockets failed to yield range data because they are concavities, and regions of the face parallel to the light plane are still challenging to capture. These regions include the middle of the forehead, the bridge of the nose, the tip of the nose, the chin, a small part of the cheeks near the nose, and the lips. Temporal processing alleviates this problem to some degree as these points do have at least part of the laser stripe passing over them.

In practice, we found many 3D points are acquired that are not helpful for facial recognition like parts of the neck, the shoulders, and the ears. We made a cropping algorithm based on the location of the nose and a distance measure to each point. The location of the nose is assumed to be in the first frame where a laser stripe image is visible. The Euclidean distance between the $(y$, $z$ ) coordinates of the nose and all the other face points is calculated. A histogram of these distances (Figure 9 (a)) is typically bimodal if objects other than the face are present in the output. The histogram is smoothed, and an ad hoc threshold is identified in the valley between smoothed peaks. All of the points in the larger depth mode are cut off, yielding a cropped image.

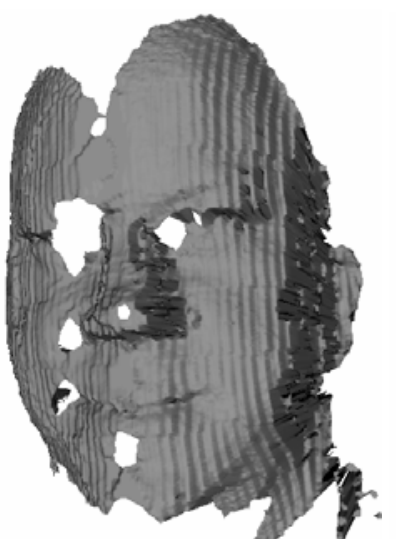

(a)

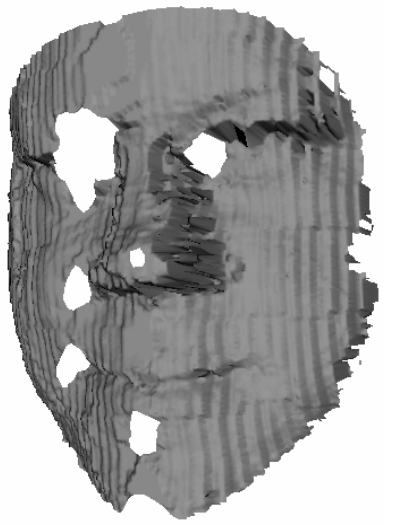

(b)
Figure 8: (a) an example of a 3D image of a human subject's face. (b) The same face with the ears and neck cropped.
Most models have some large holes due to concavities of the face or the flatter regions of the face. Three more examples of the resulting 3D face models (Figure 10) have some holes in the eye and around the nose. These are mostly unavoidable with our current setup, but future work will attempt to fill these holes.

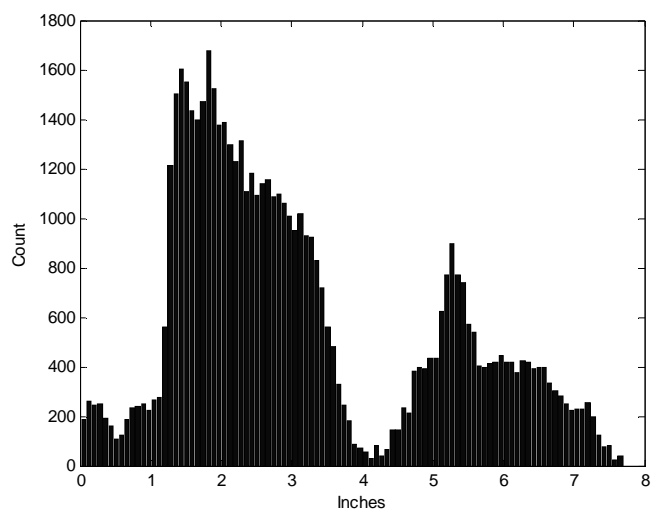

(a)

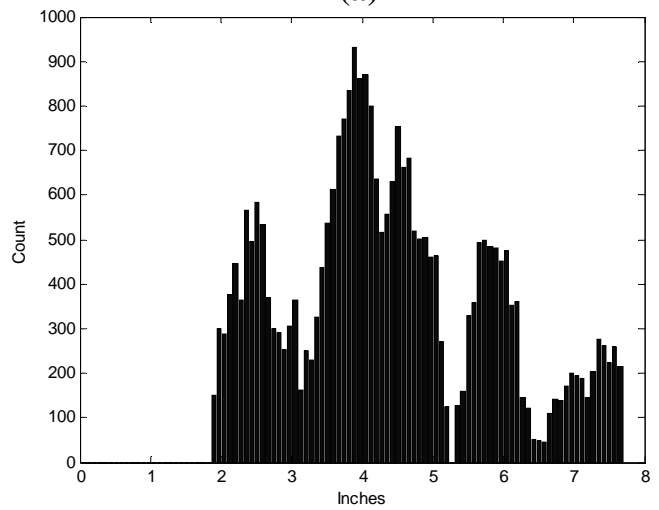

(b)

Figure 9: (a) Histogram of the Euclidean distance of the YZ-plane from the nose. (b) The difference between the smoothed histogram $(20$ point sliding window) and the original histogram. The peak location corresponds to a distance of 4 inches form the nose tip, which is a reasonable cutoff threshold.

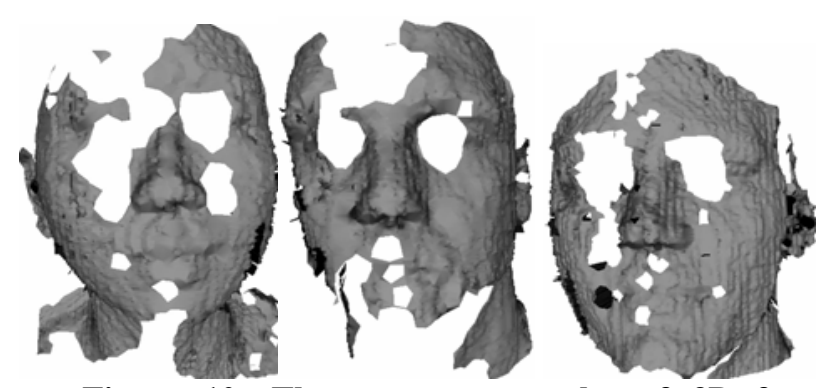

Figure 10: Three more examples of $3 D$ face models made using our setup. 


\subsection{Possible Difficulties: Pitch, Yaw, and Roll}

Human subjects can change their head position and orientation as they intersect the light screen. This can be seen in shifts along the $\mathrm{x}$-axis, $\mathrm{y}$-axis, and $\mathrm{z}$-axis as well as changes in the pitch, yaw, and roll of the subject's head. A shift along the z-axis happens in all cases because the subject has to move through the light screen. This shift is tracked by the side camera, and a shift in the y-axis could also be tracked using the images from the side camera. We assume there to be minimal shift in the $y$-axis based on experimental observations, so we do not track a shift in the y-axis. We also assume zero pitch, yaw, and roll change, and we will address these changes in future work.

\section{Biometric Matching}

The end use of our 3D face scanner is biometric matching. A variety of measures have been developed to determine the similarity of two 3D objects. A popular approach in 3D face recognition is to calculate and tabulate the alignment error obtained using the Iterative Closest Point (ICP) [27] algorithm and to assume that mismatched identities will yield a large alignment error. Thus, the alignment error is a matching score where smaller values indicate a closer match.

Identification and verification are the two tasks available in biometric systems. Both tasks compare an incoming probe template (e.g., a 3D face image) to an existing database of gallery templates. Identification tasks match the probe against every template in the gallery and return the identity of the best match. A verification task matches the probe only against those templates matching an identity claim accompanying the probe. Our experiment was an identification experiment.

In our case, we used ICP [27] to compute a match score, namely the alignment error (lower values indicate better matches). ICP iteratively calculates the least squares fit of two 3D models, and after each iteration, one of the models is translated and rotated such that the error for the least squares fit decreases. This process continues until the change in the least squares fit is below a predefined tolerance level. The final alignment error is the matching score. Figure 11 shows the result of an ICP match of two models from the same subject. As one can see, one face fits very well into the other, and the only major problem areas are near parts of the face prone to having causing holes during the $3 \mathrm{D}$ imaging like the eyes, nose, and forehead.

We ran an initial experiment with 13 subjects, taking 4 images of each subject. We then ran ICP on these images using 1 image of each subject as a gallery image and the other 3 as probe images. We had a gallery set of 13 images and a probe set of 39 images. With this setup, we achieve $92.3 \%$ correct rank one recognition (Figure 12).

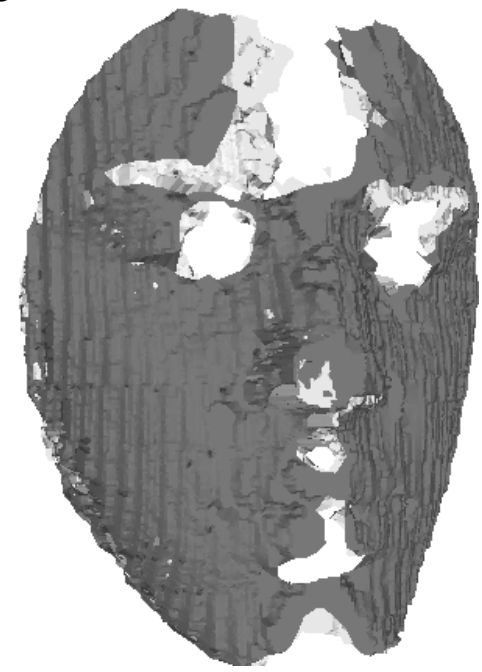

Figure 11: Two overlapping models using ICP alignment.

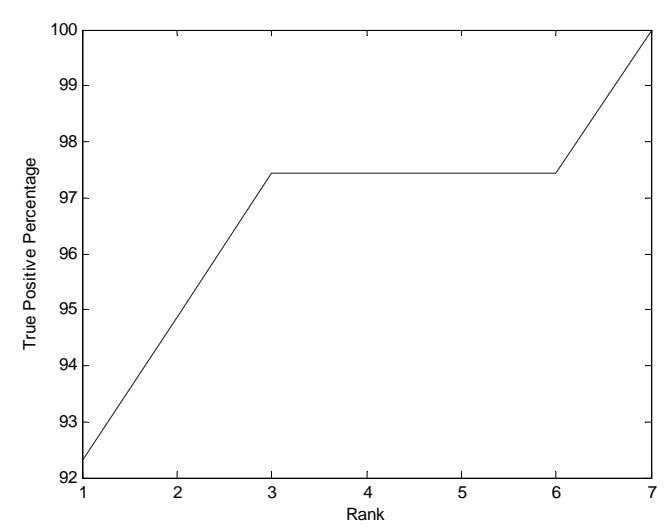

Figure 12: Shows the resulting true positive at each rank position.

\section{Conclusion}

We presented a system for 3D facial acquisition using a static light screen and a moving object. By processing in the temporal domain, we found we could build a very dense 3D image of a person's face. The resulting small-scale 3D matching experiment suggests that our technique is good enough to be used for facial recognition, but to validate this claim, a larger image set will be required.

Our technique allows a subject to walk through the sensor naturally, which is its major advantage. The quality of this technique only depends on the camera's resolution and frame rate, and we expect to achieve the quality of commercially available face imagers by upgrading the cameras. 
In the future, we will collect more data of human subjects to produce more accurate matching results. We plan to apply improvements to temporal domain processing. We also plan to modify the light screen by angling two stripes and reconstruct a 3D image from a single camera. This will hopefully eliminate some of the holes unavoidable in the current set-up. We have begun to develop software to track pitch, yaw, and roll, and we will integrate these changes in the 3D model.

\section{Acknowledgements}

Thanks to Chris Boehnen for his help in making a composite mesh image.

\section{Bibliography}

1. F.W. DePiero, and M.M. Trivedi, "3-D Computer Vision using Structured Light: Design, Calibration, and Implementation Issues." Advances in Computers (43), 1996, Academic Press, pp.243-278.

2. K. Araki, Y. Sato, and S. Parthasarathy. "High speed rangefinder.” In SPIE vol 850: Optics, Illumination, and Image Sensing for Machine Vision, pages II-184-II-188, 1987.

3. T. Kanade, A. Gruss, and L. Carley. "A very fast VLSI rangefinder." In IEEE International Conference on Robotics and Automation, pages 1322-1329, 1991.

4. G. Medioni and J. Jezouin. "An implementation of an active stereo range finder.” In Optical Society of America Technical Digest Series vol. 12, Tropical Meeting on Machine Vision, pages 34-51, 1987.

5. P. Saint-Marc, J. Jezouin, and G. Medioni. "A versatile PC-based range finding system.” IEEE Transactions on Robotics and Automation, 7(2):250-256, 1991.

6. J. Davis and X. Chen. "A laser range scanner designed for minimum calibration complexity.” In Third International Conference on 3D Digital Imaging and Modeling, 2001.

7. K. Pulli, H. Abi-Rached, T. Duchamp, L. Shapiro, and W. Stuetzle. "Acquisition and visualization of colored 3D objects.” In ICPR, pages 11-15, 1998.

8. A. Griesser, T.P. Koninckx , and L. Van Gool. "Adaptive real-time 3D acquisition and contour tracking within a multiple structured light system.” 12th Pacific Conference on 6-8 Oct. 2004 Page(s):361 - 370.

9. D. Lanman, D. Crispell, and G Taubin. "Surround Structured Lighting for Full Object Scanning.” 3-D Digital Imaging and Modeling, Sixth International Conference on 21-23 Aug. 2007 Page(s):107 - 116.

10. J. Jalkio, R. Kim, and S. Case. "Three dimensional inspection using multistripe structured light.” Optical Engineering, 24(6):966-974, 1985.

11. J. Park, G.N. DeSouza, A.C. Kak, "Dual-beam structuredlight scanning for 3-D object modeling.” 3-D Digital Imaging and Modeling, Third International Conference on 28 May-1 June 2001 Page(s):65 - 72.

12. Y.F. Wang, A. Pandey. "Interpretation of 3D structure and motion using structured lighting." Interpretation of
3D Scenes, Workshop on 27-29 Nov. 1989 Page(s):84 90.

13. D.J. Park and J.H. Kim, "3D hand-eye robot vision system using a cone-shaped structured light for the SICEICASE International Joint Conference 2006 (SICEICCAS 2006)”. SICE-ICASE, 2006. International Joint Conference Oct. 2006 Page(s):2975 - 2980.

14. K. L. Boyer, and A. C. Kak, "Color-encoded structured light for rapid active ranging," IEEE Transactions on Pattern Analysis and Machine Intelligence. Volume , Issue 1. January 1987.

15. Y. Zhang, R. Lu, Y. Fei, and X. Chen, "Multi-parameter measurement of complex part based on color encoded structured light.” Information Acquisition, International Conference on 21-25 June 2004 Page(s):319 - 322.

16. L. Zhang, B. Curless, and S. M. Seitz. "Rapid shape acquisition using color structured light and multi-pass dynamic programming.” In IEEE 3D Data Processing Visualization and Transmission, 2002.

17. X. Gu, S. Zhang, P. Huang, S.T. Yau, and R. Martin. "Holoimages." ACM Symposium on Solid and Physical Modeling, 2006, Pages 129 - 138.

18. S. Lee, J. Choi, D. Kim, J. Na, and S. Oh, "Signal Separation Coding for Robust Depth Imaging Based on Structured Light.” Robotics and Automation, Proceedings of the 2005 IEEE International Conference on 18-22 April 2005 Page(s):4430 - 4436.

19. R.B. Fisher, A.P. Ashbrook, C. Robertson, and N. Werghi, "A low-cost range finder using a visually located, structured light source." 3-D Digital Imaging and Modeling, Second International Conference on 4-8 Oct. 1999 Page(s):24 - 33.

20. N. Borghese, G. Ferrigno, G. Baroni, A. Pedotti, S. Ferrari, and R. Savare. "Autoscan: A flexible and portable 3D scanner.” IEEE Computer Graphics and Applications, 18(3):38-41, 1998.

21. B. Curless and M. Levoy. "Better optical triangulation through spacetime analysis.” In ICCV, pages 987-994, 1995.

22. J. Davis, D. Nehab, R. Ramamoorthi, and S. Rusinkiewicz, "Spacetime stereo: a unifying framework for depth from triangulation." Transactions on Pattern Analysis and Machine Intelligence On page(s): 296- 302, Volume: 27, Issue: 2, Feb. 2005.

23. H. Tomita, S. Yoda, K. Sato, and K. Chihara, "Range finding with simultaneous projection of modulated patterns." Proceedings of the 34th SICE Annual Conference. International Session Papers 26-28 July 1995 Page(s): 1449 - 1452.

24. O. Hall-Holt and S. Rusinkiewicz. "Stripe boundary codes for realtime structured-light range scanning of moving objects.” In ICCV, pages 359-366, 2001.

25. R. Fisher and D. Naidu. "A comparison of algorithms for subpixel peak detection.” In Proc. 1991 British Machine Vision Association Conf, pages 217-225, 1991.

26. Barber, C. B., D.P. Dobkin, and H.T. Huhdanpaa, "The Quickhull Algorithm for Convex Hulls," ACM Transactions on Mathematical Software, Vol. 22, No. 4, Dec. 1996, p. 469-483.

27. P.J. Besl and N.D. McKay. "A method for registration of 3D shapes,” IEEE Trans. Patt. Anal. Machine Intell., vol. 14, no. 2, pp. 239-256, 1992. 